\title{
Discovery of Small Molecule Drugs Targeting the Biogenesis of microRNA-155 for the Treatment of Systemic Lupus Erythematosus
}

\author{
Kamal Azzaoui*, Marcel Blommers, Marjo Götte, Kaspar Zimmermann, He Liu, and Heinz Fretz
}

\begin{abstract}
Systemic lupus erythematosus (SLE) is an autoimmune disease that often leads to functional disorder in multiple organs, most often with symptoms related to skin lesions, cardiovascular disease and kidney damage. Although significant efforts have been made to find efficient therapies, it still remains uncured. Furthermore, the current therapy is often associated with adverse side effects and leads to a high economic burden for society. At Saverna Therapeutics, in collaboration with our partners, we initiated a lead discovery program that aims to modulate the biogenesis of miR-155. This non-coding RNA is upregulated in SLE patients and SLE mouse models. We used our drug discovery platform based on iterative fragment-based screening by nuclear magnetic resonance (NMR) and machine learning to identify ligands of pre-miR-155. After several iterations and chemical modifications, we have identified compounds that show structure-activity relationships in cellular assays. These inhibitors reduced the level of miR-155 as well as its associated inflammatory protein TNF $\alpha$ whereas the cells remained viable during exposure of the compounds.
\end{abstract}

Keywords: FBS · Machine learning $\cdot$ microRNA $\cdot$ NMR $\cdot$ SLE

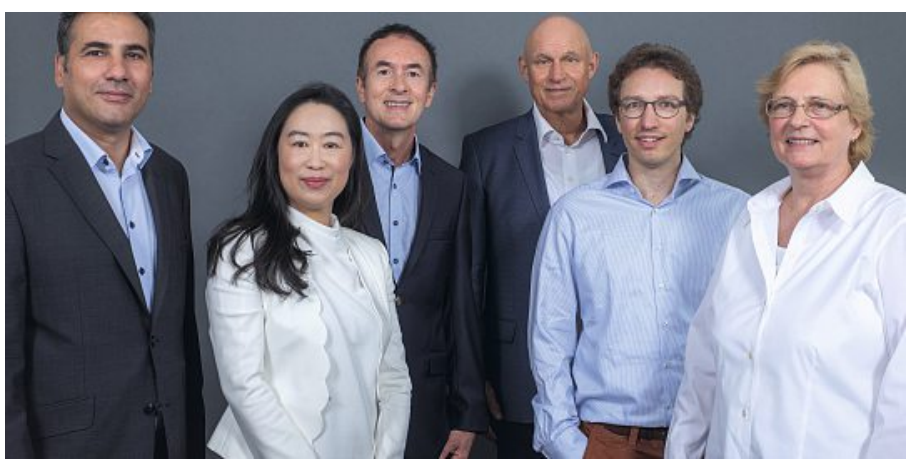

Dr. Kamal Azzaoui: CEO/co-founder, Dr. He Liu: consultant cell biology, Dr. Marcel Blommers: CSO/co-founder, Dr. Heinz Fretz: director medicinal chemistry, Dr. Kaspar Zimmermann: NMR project manager, Dr. Marjo Götte: director cell biology/co-founder.

Kamal Azzaoui received his $\mathrm{PhD}$ in computational chemistry from the University of Orléans followed by two postdocs at McGill University and Georgetown University. He also holds a Master for Advanced Science (MAS) from the Swiss Institute for Translational and Entrepreneurial Medicine (sitem-insel, University of Bern). He worked for more than 15 years in pharmaceutical industry in charge of data analysis, data modeling, drug design using machine learning, cheminformatics and molecular modeling tools. Since 2017, he is the CEO and co-founder of Saverna Therapeutics, a Swiss startup aiming to discover drugs for non-coding RNAs targets.

Marcel Blommers is an expert in drug discovery for RNA targets. After completing his $\mathrm{PhD}$ on nucleic acids (Prof. Hilbers, Nijmegen) and a postdoc (Prof. Kaptein, Utrecht), he came to Ciba-Geigy in 1992, continued at Novartis, to explore the use of
NMR in pharmaceutical research. NMR became a recognized method in drug discovery. In 1998, he was a pioneer in lead finding for the viral RNA target TAR. He contributed to the development of antisense and siRNA oligonucleotide drugs and recently to the RNA targeting drug Branaplam. He co-founded Saverna Therapeutics in 2017 and is the company's CSO.

Marjo Götte received her $\mathrm{PhD}$ in Microbiology from the University of Helsinki. After a postdoc period at Ciba-Geigy she worked as a senior scientist at the University of Zurich, studying regeneration of neurons. Thereafter she spent 16 years at Novartis with responsibility to coordinate the activities in early drug discovery, design and run the cellular assays. Her expertise lies in cellular screening especially in high-content screening. In 2017, she co-founded Saverna Therapeutics and she is responsible for the cellular assays to test the activity of the hits identified in screening.

Kaspar Zimmermann received his $\mathrm{PhD}$ in Chemistry from the University of Basel. From his studies he gained broad experience in nuclear magnetic resonance (NMR) spectroscopy on small molecules as well as on new methods for protein structure determination. He joined Saverna Therapeutics in 2019 as a project manager in NMR spectroscopy and is responsible for NMR screening, data interpretation and design and implementation of new methods.

He Liu holds a PhD in Biomedical Sciences at the University of Bern, Switzerland and has a broad research experiences in autophagy, cancer, metabolism, adult stem cells and immunology. Her research in autophagy revealed a novel tumor suppressive function of ATG5 in melanoma and has been awarded as "The Best Doctoral Thesis in Dies Academicus" in 2014. In 2019, she enrolled in the 'MAS Translational Medicine and Biomedical Entrepreneurship' program at Sitem-Insel to gain entrepreneurial mindset. She took the role as consultant at Saverna in 2020 and is involved in $\mathrm{R} \& \mathrm{D}$ project plan and research collaborations. 
Heinz Fretz studied Biochemistry and Organic Chemistry and received his $\mathrm{PhD}$ in Organic Chemistry from the University of Zurich. After postdoctoral studies at Harvard University and University of Zurich, he started his industrial career in drug discovery at Ciba-Geigy. During his 27 professional years as medicinal chemist in various positions at Novartis, Genomics Institute of the Novartis Research Foundation (GNF), Axovan, Actelion, and Idorsia, he gathered vast experience in drug discovery and discovery technologies. In 2019, he joined the Saverna Therapeutics team as Director of Chemistry.

\section{Business Case, Context, Motivation}

Systemic lupus erythematosus (SLE) is a chronic autoimmune disease affecting primarily females of childbearing age with a gender bias of 9:1, partially attributed to estrogen receptor-1 and to other unknown immunomodulatory genes on the X chromosome. ${ }^{[1]}$ Various genetic and epigenetic factors result in the heterogeneous clinical presentations caused by immune complex-induced inflammation in multiple tissues and organs, such as skin, kidney, heart, lung and brain. ${ }^{[2]}$ The estimated incidence of SLE in west European countries ranges from 2.2 to 5 per 100,000 inhabitants and the prevalence from 20.5 to 91 per 100,000. According to the Lupus Foundation of America, 1.5 to 2 million Americans have some form of lupus. Worldwide about 5 million people are estimated to suffer from lupus. The average direct annual costs per patient were 8274 USD in Canada in 2010. [3]

Pathogenesis of SLE is complex and multifactorial involving both the innate immune system that initiates the inflammatory cascade leading to disease onset, and the adaptive immune system that leads to the ongoing activities of SLE. Multiple genes known to contribute to the disease susceptibility have been described, among which, the genes of the major histocompatibility complex (MHC) have been most extensively investigated. With an exception of C1q deficiency, a minimal number of four susceptibility genes are required for the development of SLE, thus explaining the polygenic inheritance of the disease. Environmental factors, such as Epstein-Barr virus, ultraviolet light, cigarette smoke and certain drugs are known to facilitate the development and/ or progression of SLE in predisposed individuals. Loss of immune tolerance and development of autoimmunity, evidenced by defective $B$ cell suppression, excess in T cell help and shifting of $\mathrm{T}$ helper 1 (Th1) to Th2 immune responses lead to hyperactivation of B cells and production of pathogenic autoantibodies. The important role of B cells in the pathogenesis of SLE is not only reflected by their abilities to produce autoantibodies, but also their ability to present autoantigens to T cells via B cell receptor as well as cytokine secretion and modulation of the activity of dendritic cells (DCs). Impaired clearance of apoptotic cells and formation of immune complexes lead to defective immune regulation, representing important contributors to the development of SLE. ${ }^{[4-6]}$

The heterogeneity of the disease manifestations and the driving causes of SLE result in the challenges to treat the disease and to advance the current treatment strategies that include systemic corticosteroids, antimalarial drugs, immunosuppressants and nonsteroidal anti-rheumatic drugs, either applied alone or in combination, depending on the affected tissues or organs and disease classification that has been discussed intensively elsewhere. ${ }^{[4]}$ Although the mortality of SLE has been largely reduced with a 15-year survival of 85-95\%, effective management of SLE treatment is often associated with severe drug-induced toxicity leading to deterioration of organ function and infections, in particular in patients with refractory disease and/or lupus nephritis. ${ }^{[4]}$ Thus, an unmet medical need to develop safer and more efficacious therapies against SLE still remains. A breakthrough in SLE treatment is belimumab (Benlysta ${ }^{\circledR}$, GlaxoSmithKline), a fully humanized monoclonal antibody that binds to soluble B cell activating factor, leading to reduction in circulating CD20+ B cells. ${ }^{[7]}$ Up to now, Belimumab is the only approved drug applicable for the treatment of SLE by the U.S. Food and Drug Administration. More than 600 clinical studies for SLE have been initiated, and at present 47 studies are recruiting participants in different clinical trials ( 2 in early phase I, 11 in phase I, 24 in phase II, 6 in phase III and 4 in phase IV). Similar trials with the drug action aiming at deletion or reduction of B cells have also been initiated with e.g. obinutuzumab, an anti-CD-20 antibody (NCT02550652) and many others with related information available at www.clinicaltrials.gov. Despite the improvement of SLE therapy using biologicals, general problems associated with antibody treatment still remain unsolved, for instance toxicity, inconvenient delivery as well as high costs in production, thus an unaffordable cost for patients and huge economic burden for society. In contrast, low molecular weight (LMW) drugs, which require lower development and production costs and can be administered orally, are an attractive alternative to biologicals.

Saverna Therapeutics AG was founded in late 2017 by four scientists with extensive experience in the pharmaceutical industry. The mission of the company is to discover and develop safe, orally active small molecules that target non-coding RNA biology to treat diseases with unmet medical need. MicroRNAs (miRNAs), a class of endogenous small non-coding RNAs have been found to play essential roles in regulating many physiological and pathological processes. Through regulation of the stability and translation of mRNAs involved in the pathogenesis of SLE, these miRNAs represent an attractive collection of novel drug targets. Our experience in RNA biology is exemplified by our previous contribution to one of the first RNA-targeting LMW drug candidates, LMI070/Branaplam, that is in clinical trials to treat patients with spinal muscular atrophy (NCT02268552). Using our innovative drug discovery platform consisting of integrated fragment-based screening (FBS) by nuclear magnetic resonance (NMR), in silico machine learning (ML) and cellular assays, we have discovered hit molecules that are able to inhibit biogenesis of miR-155. This RNA is abundant in SLE patients and is known to play essential roles in the pathogenesis of SLE from a body of in vitro and in vivo data. ${ }^{\left[{ }^{8-10]}\right.}$ With the help of our scientific advisors in the field of rheumatology, NMR, and RNA, we are encouraged to validate the lead molecule and to translate our findings into a clinical setting aiming at developing the first LMW drug available on the market for treatment of SLE. The commercialization of such a molecule will be achieved through the establishment of a partnership deal with a pharma partner for development of the drug and a licensing deal for eventual sales. We aim to enter partnership deals once the full clinical trial application (CTA)requiring data package is completed. The drug will be developed to the stage where preclinical efficacy, pharmacokinetic (PK) and initial toxicity have been evaluated.

\section{Solution, Scientific Approach}

miRNAs, whose genes are often located within intronic regions of a genome, are located in a clustered manner and can be transcribed either as one primary transcript by a common promoter or transcribed individually using different promoters. Primary transcript of a miRNA, i.e. pri-miRNA is cleaved by Drosha in the nucleus, resulting in a small hairpin RNA, referred to as pre-miRNA that is released and exported to the cytoplasm by Exportin-5. In the cytoplasm, pre-miRNA is cleaved by RNase III, called Dicer, into a 22 nt-long duplex RNA. Other double-stranded RNA-binding proteins assist in this process which results in two mature miRNA strands. Depending on their thermodynamic properties, one of the two strands is preferred to incorporate into the RNA-induced silencing complex (RISC), which contains the Argonaute (Ago) family of proteins responsible for the execution of the inhibitory function of miRNA on its target mRNA ${ }^{[11]}$ (Fig. 1). 


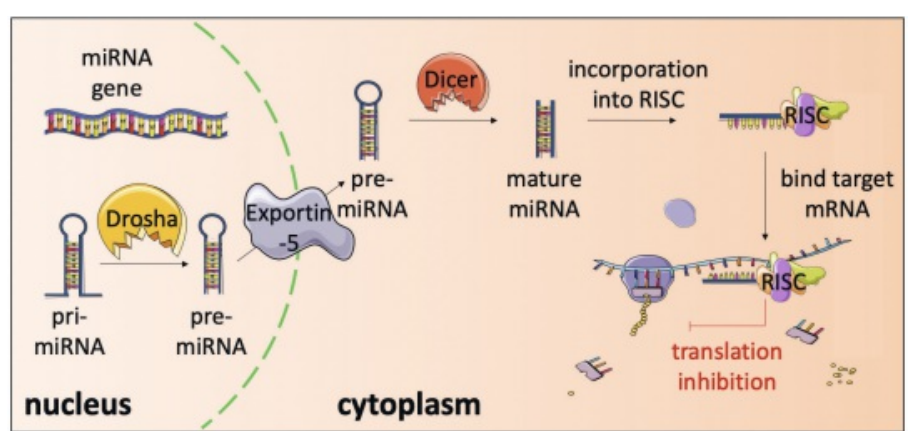

Fig. 1. Overview of the formation and function of miRNA in a cell. miRNA is transcribed by RNA polymerase II as pri-miRNA that is cleaved by Drosha in the nucleus to form pre-miRNA. Pre-miRNA is then exported from the nucleus to the cytoplasm via the nucleocytoplasmic shuttle, Exportin-5. In the cytoplasm, pre- miRNA is further processed to mature miRNA; and one of the strands of the mature miRNA is incorporated into RISC, allowing miRNA to execute its inhibitory function on translation of the target mRNA. A more detailed secondary structure of pre-miR155 is shown in Fig. 3. Smart arts (Smart Servier Medical Art, https://smart.servier.com) with own modifications were used to illustrate this figure.

Silencing of mRNA transcripts at the posttranscriptional level by miRNAs belongs to one of the mechanisms in epigenetic regulation of genes. So far, more than 2000 miRNAs have been identified in humans and they may regulate the expression of approximately $60 \%$ of all human protein-coding genes as shown by computational prediction. ${ }^{[12]} \mathrm{miR}-155$ is one of the most widely recognized miRNAs known to be involved in autoimmune diseases through its crucial activities in innate and adaptive immunity. The expression of miR-155 is increased upon infection or injury and decreased by encountering anti-inflammatory cytokines, resolvins and glucocorticoids. For instance, miR-155 regulates polarization of macrophages in favor of a pro-inflammatory activation rather than an anti-inflammatory and repairing phenotype. It has been shown that deficiency of miR-155 reduces the levels of pro-inflammatory cytokines both in murine and in human macrophages. ${ }^{[13]}$ In DCs, the crucial cell type in shaping the $\mathrm{T}$ cell repertoire and determining the nature of T cell response, miR-155 is known to regulate DC activation through repression of Src homology 2 domain-containing inositol-5'-phosphatase 1 (SHIP-1) and suppressor of cytokine signaling-1 (SOCS-1) in a similar manner as in macrophages. Experimentally, it has been shown that overexpression of miR-155 alone is sufficient to break selftolerance and promote a CD8- mediated autoimmune response in a diabetes model. ${ }^{[14]}$ In adaptive immunity, miR-155 is known to regulate B cell proliferation through SHIP and CCAAT enhancer-binding protein $\beta$ (C/EBP $\beta)$. In addition, the formation of the germinal center (GC), a structure essential for antibody production, is positively supported by miR-155 via targeting PU.1, which reduces the expression of Pax5, an inhibitor of B cell activation. This enables plasma cell differentiation together with the expression of genes involved in $\mathrm{B}$ and $\mathrm{T}$ cell interactions, facilitating antibody production. Multiple studies have shown that miR-155 is upregulated in B cell-associated diseases including B cell malignancies and autoimmune diseases. ${ }^{[15]}$ Finally, miR-155 has been proven to be a regulator of $\mathrm{T}$ cell differentiation. Taken together, the mediator function of miR-155 in the human immune system, which is impaired in autoimmune diseases, including SLE, shows the rationality of targeting miR-155 in the fight against SLE. In fact, clinical trials already attempted to target miRNAs with oligonucleotides, e.g. anti-miR-122 in hepatitis $\mathrm{C}$ infection ${ }^{[16]}$ and a mimic of the tumor suppressor miRNA-34 in cancer.[17]

To fight against SLE, we have developed a unique methodology that combines FBS by NMR and ML to target miR-155. This RNA has been shown to be abundant in SLE patients. Its downregulation by inhibition of its maturation prevents secretion of proinflammatory cytokines. ${ }^{[18-20]}$ General steps in drug discovery and development processes of the preclinical phase include target identification and validation, hit to lead, lead optimization, candidate profiling and finally preclinical development. A suitable pre-miR-155 construct and small molecular fragments library were used to screen hits, compounds that bind to pre-miR-155 by NMR. The binding site of hits, as determined by NMR, is in the region where dicer cleaves pre-miR-155 in the process of maturation. Based on these findings, ML models were created and applied to molecular supplier catalogs to buy larger molecules or alternatively to synthesize larger molecules together with our chemical partner SpiroChem. The chemical classes that show structure-activity relationships were prioritized for suitable cellular assays and animal model testing.

FBS is a widely used approach to find hits and/or lead molecules that may initially have weak to moderate affinity for binding to the target of interest. Sequential steps are required to obtain medium to strong binders by growing or combining these small chemical fragments. Traditional high throughput screening (HTS) approach has been replaced in some companies by FBS due to the fact that FBS requires significantly fewer compounds for screening and synthesis and it often results in a higher success rate in generating chemical series harboring lead-like properties. The most robust applied biophysical detection method in FBS is NMR that is sensitive enough to detect the binders with low affinity. Since the chemical compounds and the target RNA are observed directly, the chemical integrity of test compounds and the folding of the RNA are confirmed during the screening experiment. The number of false positive hits is therefore extremely low. Furthermore, NMR also provides structural information about the binder-target complex and is able to help evolve the fragments towards high affinity larger molecules. ${ }^{[21]}$ We have applied FBS by NMR to find hits and/or leads of a novel class of molecular targets, pre-miRNAs.

Within Saverna, simulation and machine learning are used in all phases of drug discovery by providing tools that can improve discovery and decision making, increase lead finding success rates and dramatically reduce costs. We used information from FBS to train ML models of binders and non-binders, and used them to grow the fragments into larger molecules to obtain more efficient ligands with high affinity for pre-miR-155. In addition, we have also used other ML models, for instance, those for cell permeability, drug likeliness as well as solubility to further refine our candidate lead series with the least liabilities to enter clinical studies. We have more than $50 \mathrm{ML}$ models that have been trained with large amounts of data to predict the success of a hit-to-lead drug discovery process. Our inhouse CRIBOSAR package is a cheminformatics toolbox based on deep learning, classifications and regressions (Fig. 2). Adding to the ML approach, our vast experience in the selection of fragments and filtering out compounds with liability allows us to choose or design compounds with higher chance to succeed in clinical trials. ${ }^{[22,23]}$

Initially, we screened the iNEXT library. This is a poised fragment library that was designed for a handy follow-up of compound selection and synthesis. Examination of the library by cheminformatics tools ensured its chemical diversity. Quality control of the library by NMR has been performed by Saverna and by Prof. Harald Schwalbe's lab (University of Frankfurt) to guarantee that only soluble and stable compounds will be used for screening. ${ }^{[24]}$ The first batch of the pre-miRNA-155 to be used in the FBS campaign has been synthesized in Prof. Frédéric Allain's lab (ETH Zurich). A total of 190 fragments in 12 mixtures have initially been screened by Saverna using NMR in the presence of miR-155 at Dr. Daniel Häussinger's lab (University of Basel) using a Bruker $600 \mathrm{MHz}$ instrument equipped with a cryoprobe and a sample changer. Six primary hits with clear effects and six with weak effects were observed. After the primary screen in mixtures, 


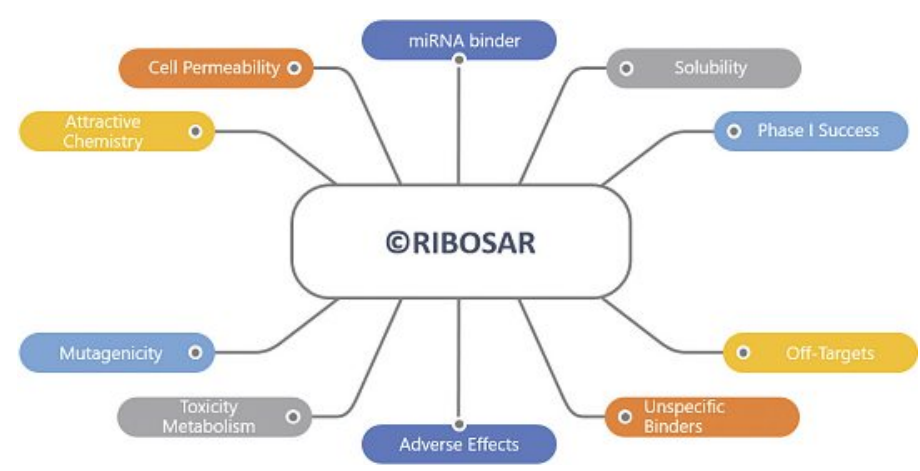

Fig. 2. Workflow of our ML tool for drug discovery. More than $50 \mathrm{ML}$ models trained with large amounts of public data are applied to predict the success of a hit-to-lead project and beyond. These models are also used to design and review libraries, annotation of compounds in collections and to prioritize hits.

the hits were validated as single compounds in the presence of pre-miR-155 during which two hits were confirmed. Selectivity study by NMR using other RNAs and miRNAs showed that only one hit was specific to miR-155, indicating the feasibility of our method to find compounds that bind pre-miR-155. An example of the proof of binding of a fragment hit from the first FBS campaign of pre-miR-155 is shown in Fig. 3. Later, it could be shown that several of our hits were cell-permeable and showed significant down regulation of miR-155 and of cytokines.

Taken together, we have proved the feasibility of targeting premiRNAs with a novel approach combining FBS by NMR and ML as a time-saving and cost-efficient means applicable in early phase of drug discovery. Our approach can be adapted to any miRNA as long as its role in disease is demonstrated. The FBS approach requires a small number of compounds to be screened (500-1000) and can be more efficient than the HTS approach which requires a dedicated infrastructure, a large number of compounds (100k$1 \mathrm{Mio})$, and a time-consuming validation of hits.

\section{Product and Outlook}

The initial goal is to identify a compound and to develop it into a drug for the treatment of SLE. Over-expression of miR-155
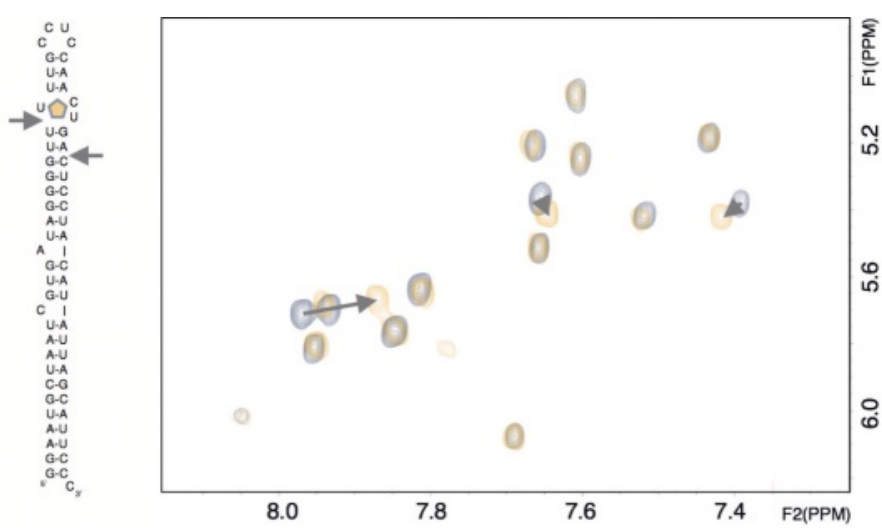

Fig. 3. Example of the validation of a fragment that binds to premiR-155. Following the primary screen in mixtures, RNA was measured as a single compound in the presence of the fragment hit. The 2D NMR spectra of the RNA in the absence (grey contours) or the presence (orange contours) of the compound are shown. With three resonances of the RNA, a clear chemical shift perturbation caused by binding of the hit was observed, as indicated by arrows. These three signals correspond to the nucleotides of the bulge in the secondary structure of pre-miR155 shown on the left. Chemical shift mapping thus indicates a specific binding of the fragment to this region that is close to the dicer cleavage site. The binding of the fragment is indicated with a small pentagon; and the arrows show where dicer cleaves. has been reported in the majority of SLE patients, equivalent to a global market of over 500,000 patients/year. Current treatments are either ineffective, expensive, and/or associated with substantial side effects, leaving $80 \%$ of patients suffering from severe symptoms. SLE is a disease with high unmet medical need, highlighted by the SLE drug market projection of \$1-3 billion by 2025 .

We demonstrated that selective binders to the untapped target, pre-miR-155 can be identified with our unique approach (Figs 2 and 3). The compounds discovered and their follow-up compounds have potential to become the first example of a cost-efficient anti-miR-155 LMW drug that can be administrated orally. SLE as a rare disease is ideal to be used as a proof of concept. By targeting miR-155 maturation we are able to suppress two main pathways that are hyperactive in the pathogenesis of SLE, i.e. the innate and the adaptive immunity, thus increasing the efficacy of the drug. Although numerous clinical trials for lupus have been initiated already since 1990 to overcome its high economic burden of 12.4 billion USD for the 1.5 million lupus patients in America, no cost-effective drugs have been developed for lupus until now. In contrast to other approaches that are often based on antibodies or proteins, which need to be injected, are expensive to produce and usually target only one component in the several pathways that are known to be dysregulated in lupus, Saverna aims for orally available small molecules that are cost-effective and are able to block both upregulated pathways in lupus patients, the innate and the adaptive immunity pathways.

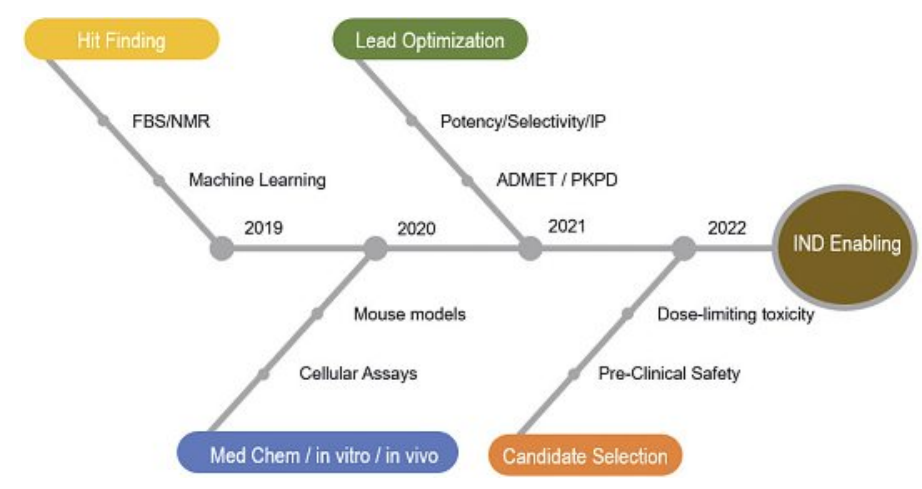

Fig. 4. Roadmap of our drug discovery process. The initial hit finding has been successfully finished and we are facing the in vitro and in vivo validation of our hit compounds at the moment. In the next two years, we will shift our focus on lead optimization and candidate selection, allowing investigational new drug (IND) application.

We have started a consortium named BUTTERFLY that is supported by a Eurostars' grant, in collaboration with SpiroChem (Basel, Switzerland) and Redoxis (Lund, Sweden), with the aim to synthesize novel compounds and to test these compounds in cells and later in vivo. It is planned to validate our lead drug in disease-relevant animal models at Redoxis and to further profile it to enter preclinical testing. With our academic partners (University of Basel, ETH Zurich supported by an Innosuisse grant), we aim to deeply understand the mechanism through which small molecules bind pre-miRNAs by NMR, surface plasmon resonance (SPR) and cellular assays.

After first showing convincing inhibition of inflammation in cells, we have started in vivo studies recently. Preliminary results show a promising trend in TNF $\alpha$ reduction in a mouse model for a 'non-optimized' chemical series. We are planning to file a Patent Cooperation Treaty (PCT) patent of the chemical series to protect our intellectual property and start pre-clinical safety studies. We aim to have an Investigational New Drug (IND) application ready by the end of 2022 (Fig. 4). By then, out-licensing opportunities will be offered worldwide to assure a fast revenue generation that will be invested in other projects that have been already started at Saverna, 
in particular in the field of inflammation, infectious diseases as well as in cancer. We collaborate with renowned academic institutions worldwide and offer services to organizations that want to use our technology and expertise to develop medicines with the goal of significantly reducing time and drug discovery costs.

\section{Saverna \\ Therapeutics AG}

Saverna Therapeutics AG was founded in November 2017 by four senior lead scientists, who have come together to leverage their complementary expertise in a specialized and validated drug discovery platform tailored for identifying RNA-targeting small molecule compounds.

\section{Key Figures}

Founding Year

November 2017

Location

Headquarter: Allschwil

Legal Form

Corporation

Founders

Dr. Kamal Azzaoui

Dr. Marcel Blommers

Dr. Marjo Götte

Dr. Jasna Fejzo

Scientific Advisors

Prof. Harald Schwalbe

Prof. Peter Wipf

Dr. Rao Movva

No of employees

Market capitalization

6

\section{Key Inventions}

Lupus Project

\section{Next Milestones}

June 2020

December 2020

January 2022

\section{Contact}

Contact Person

Address

E-mail

Website

Saverna Therapeutics AG

Office: Gewerbestrasse 24

CH-4123 Allschwil, Switzerland

Administration: Pumpmattenweg 3 CH-4105 Biel-Benken, Switzerland

\section{Acknowledgements}

We would like to thank Prof. Harald Schwalbe, Prof. Daniel Häussinger, Prof. Diego Kyburz, Dr. Andre Tiaden, Simone HänerMassimi, Prof. Frédéric Allain, Christine von Schroetter, Prof. Jonathan Hall, Artur Laski, Prof. Uyen Huynh-Do, Prof. Peter Wipf, Prof. JeanLouis Reymond, Dr. Doriano Fabbro, Dr. Rao Movva, Dr. Christian Schneider, Dr. Jasna Fejzo as well as our BUTTERFLY consortium partners, Dr. Thomas Fessard, Dr. Florent Beaufils, Dr. Christophe Salome (SpiroChem) and Dr. Malin Hultqvist, Dr. Ingrid Yao Mattisson (Redoxis). We would also like to thank our founding bodies, Eurostars (E12979) and Innosuisse (32598.1 IP-LS) for their financial support enabling the start of our lupus project as well as our investors: Viva Biotech and Roger Ganz. Purchase of the fragment library was supported by iNEXT, Grant number 653706, funded by the Horizon 2020 program of the European Commission.

Received: June 29, 2020

[1] A. V. Rubtsov, K. Rubtsova, J. W. Kappler, P. Marrack. Autoimmun. Rev. 2010, 9, 494

[2] P. L. Carreira, D. A. Isenberg, Rheumatology 2019, 58, 382

[3] A. E. Clarke, M. B. Urowitz, N. Monga, J. G. Hanly, Arthritis Care Res. 2015, $67,431$.

[4] T. Dörner, R. Furie, Lancet 2019, 393, 2344.

[5] O. Zharkova, T. Celhar, P. D. Cravens, A. B. Satterhwaite, A. M. Fairhurst, L. S. Davis, Rheumatology 2017, 56, i55.

[6] S. P. Menez, B. Essawy EI, M. G. Atta, Re Recent Clin. Trials 2018, 13, 105.

[7] M. A. Mahieu, V. Strand, L. S. Simon, P. E. Lipsky, R. Ramsey-Goldman, Lupus 2016, 25, 1122

[8] M. Khoshmirsafa, N. Kianmehr, R. Falak, S. J. Mowla, F. Seif, B. Mirzaei, M. Valizadeh, M. Shekarabi, Int. J. Rheum. Dis. 2019, 22, 458.

[9] H. R. Aboelenein, M. T. Hamza, H. Marzouk, R. A. Youness, M. Rahmoon, S. Salah, I, Abdelaziz, Growth Factors 2017, 35, 49 .

[10] S. Zhou, Y. Wang, Y. Meng, C. Xiao, Z. Liu, P. Brohawn, B. W. Higgs, B. Jallal, Q. Jia, B. Qu, X. Huang, Y. Tang, Y. Yao, J. B. Harley, N. Shen, Arthritis Rheumatol. 2016, 68, 953 .

[11] R. C. Lee, R. L. Feinbaum, V. Ambros, Cell 1993, 75, 843.

[12] R. C. Friedman, K. K. Farh, C. B. Burge, D. P. Bartel, Genome Res. 2009, 19,92 .

[13] K. Essandoh, Y. Li, J. Huo, G. C. Fan, Shock 2016, 46, 122.

[14] E. F. Lind, D. G. Millar, D. Dissanayake, J. C. Savage, N. K. Grimshaw, W. G. Kerr, P. S. Ohashi, J. Immunol. 2015, 195, 4632.

[15] D. Lu, R. Nakagawa, S. Lazzaro, P. Staudacher, C. Abreu-Goodger, T. Henley, S. Boiani, R. Leyland, A. Galloway, S. Andrews, G. Butcher, S. L. Nutt, M. Turner, E. Vigorito, J. Exp. Med. 2014, 211, 2183.

[16] R. Rupaimoole, F. J. Slack, Nat. Rev. Drug Discov. 2017, 16, 203.

[17] G. Misso, M. T. Di Matino, G. De Rosa, A. A, Farooqi, A. Lombardi, V. Campani, M. R. Zarone, A. Gullà, P. Tagliaferri, P. Tassone, M. Caraglia, Mol. Ther. Nucleic Acids 2014, 3, e194.

[18] J. Q. Chen, G. Papp, S. Póliska, K. Szabó, T. Tarr, B. L. Bólint, P. Szodoray, M. Zeher, PLoS One 2017, 12, e0174585.

[19] H. Leiss, W. Salzberger, B. Jacobs, I. Gessl, N. Kozakowsk, S. Blüml, A. Puchner, A. Kiss, B. K. Podesser, J. S. Smolen, G. H. Stummvoll, PLoS One 2017, 12, e0181015.

[20] Q. Xin, J. Li, J. Dang, X, Bian, S. Shan, J. Yuan, Y. Qian, Z. Liu, G. Liu, Q. Yuan, N. Liu, X. Ma, F. Gao, Y. Gong, Q. Liu, J. Immunol. 2015, 194, 5437.

[21] A. D. Gossert, W. Jahnke, Prog. Nucl. Magn. Spectrosc. 2016, 97, 82.

[22] K. Azzaoui,, J. Hamon, B. Faller, S. Whitebread, E. Jacoby, A. Bender, J. L. Jenkins, L. Urban, ChemMedChem 2007, 2, 874.

[23] K. Azzaoui, J. P. Priestle, T. Varin, A. Schuffenhauer, J. L. Jenkins, F. Nigsch, A. Cornett, M. Popov, E. Jacoby, in 'Data Mining in Drug Discovery', Eds. R. D. Hoffmann, A. Gohier, P. Pospisil, Wiley - VCH, Weinheim, 2013, pp. 131.

[24] S. Sreeramulu, C. Richter, T. Kuehn, K. Azzaoui, M. J. J Blommers, R. Del Conte, M. Fragai, N. Trieloff, P. Schmieder, M. Nazaré, E. Specker, V. Ivanov, H. Oschkinat, L. Banci, H. Schwalb, J. Biomol. NMR 2020, published online (https://doi.org/10.1007/s10858-020-00327-9).

License and Terms

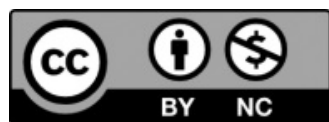

This is an Open Access article under the terms of the Creative Commons Attribution License CC BY_NC 4.0. The material may not be used for commercial purposes.

The license is subject to the CHIMIA terms and conditions: (http:// chimia.ch/component/sppagebuilder/?view=page \&id=12).

The definitive version of this article is the electronic one that can be found at https://doi.org/10.2533/chimia.2020.798 